\title{
miR-1236-3p represses the cell migration and invasion abilities by targeting ZEB1 in high-grade serous ovarian carcinoma
}

\author{
YU WANG ${ }^{1}$, SHI YAN ${ }^{1}$, XIAOLIN LIU ${ }^{1}$, WENJING ZHANG ${ }^{1}$, YINGWEI LI ${ }^{1}$, RUIFEN DONG ${ }^{1}$, \\ QING ZHANG $^{1}$, QIFENG YANG ${ }^{2}$, CUNZHONG YUAN $^{1}$, KENG SHEN $^{3}$ and BEIHUA KONG ${ }^{1}$
}

\begin{abstract}
Departments of ${ }^{1}$ Obstetrics and Gynecology and ${ }^{2}$ Breast Surgery, Qilu Hospital, Shandong University School of Medicine, Jinan, Shandong 250012; ${ }^{3}$ Department of Obstetrics and Gynecology, Peking Union Medical College Hospital,

Peking Union Medical College, Beijing 100730, P.R. China
\end{abstract}

Received January 12, 2014; Accepted February 3, 2014

DOI: $10.3892 / o r .2014 .3046$

\begin{abstract}
Ovarian cancer, particularly high-grade serous ovarian carcinoma (HG-SOC), is still the main cause of death among gynecological malignancies. However, the molecular mechanisms related to its malignant biological behavior are still unclear. Recent studies indicate that microRNAs (miRNAs) play an important role in tumor metastasis. Here, we report that miR-1236-3p expression was downregulated in HG-SOC when compared to that in normal fallopian tube tissue. Manipulation of miR-1236-3p significantly influenced the morphology, migration and invasion of ovarian cancer cell lines (A2780 and SKOV3). With dual-luciferase reporter assay, we demonstrated that miR-1236-3p binds to the 3'UTR of zinc-finger E-box binding homeobox 1 (ZEB1) mRNA, and functions as a negative regulator of ZEB1. Furthermore, we revealed that manipulation of miR-1236-3p modulates ZEB1 expression and influences expression of its downstream genes E-cadherin and $\mathrm{N}$-cadherin at both the mRNA and protein levels. We also found an inverse relationship between miR-1236-3p and ZEB1 expression in the HG-SOC tissue samples. Taken together, our results indicate that miR-1236-3p regulates ovarian cancer metastasis by directly targeting ZEB1, and it may play an important role in the diagnosis and treatment of ovarian cancer.
\end{abstract}

\section{Introduction}

Even after decades of research investments, ovarian cancer remains the most lethal gynecological malignancy. The 5-year survival rate is only $44 \%$ in the USA (1). Due to histologic and

Correspondence to: Professor Beihua Kong, Department of Obstetrics and Gynecology, Qilu Hospital of Shandong University, 107 Wenhua Xi Road, Jinan, Shandong 250012, P.R. China

E-mail: kongbeihua@sdu.edu.cn

Key words: miR-1236-3p, epithelial-mesenchymal transition, zinc-finger E-box binding homeobox 1, ovarian serous carcinoma, metastasis molecular heterogeneity, epithelial ovarian cancer (EOC) is not usually considered a single entity. EOC can be divided into four major subtypes: serous, mucinous, endometrioid and clear cell adenocarcinomas. Serous adenocarcinoma is the most common among the subtypes and consists of low-grade and high-grade carcinomas. Most ovarian cancer deaths are associated with high-grade serous ovarian carcinoma (HG-SOC) (2), which originates from the fallopian tube fimbriae according to recent studies (3-7). Metastasis is the major cause for the high mortality rate. Therefore, it is crucial to clarify the molecular mechanisms that influence the metastasis of HG-SOC.

Epithelial-mesenchymal transition (EMT) is known as a key regulatory mechanism of migration and invasion in many types of cancers including ovarian cancer $(8,9)$. EMT is a morphological change where cells switch from a polarized epithelial phenotype to a highly motile mesenchymal phenotype (10). During the process of EMT, cells lose epithelial adhesion molecules (such as E-cadherin) and acquire mesenchymal markers (such as $\mathrm{N}$-cadherin), with increased migration and invasion. Several transcriptional factors such as Twist, ZEB and Snail have been reported as inducers of EMT (11). Among these factors, the zinc-finger E-box binding homeobox 1 (ZEB1) is known to be overexpressed in ovarian cancer and may directly repress the epithelial marker E-cadherin to induce EMT (12).

MicroRNAs (miRNAs) are a class of small ( 22 nucleotides) non-coding RNAs that negatively modulate gene expression in a sequence-specific manner, and they are conserved in evolution (13). miRNAs play important roles in numerous biological processes, such as cell cycle, differentiation, proliferation, apoptosis and angiogenesis (14-16). The relationship between miRNAs and cancer was first discovered in chronic lymphocytic leukemia (17). Since then, numerous miRNAs have been found abnormally expressed in many types of cancers including ovarian cancer (18). EMT was also found to be modified by many miRNAs, such as the miR-200 family, miR-23b, miR-29b and miR-150 (19-23). In the present study, we examined the expression of miR-1236-3p in HG-SOC and normal fallopian tube tissues. We found that miR-1236-3p was downregulated in HG-SOC. We then investigated the effect of miR-1236-3p on tumor metastasis and showed that miR-1236-3p overexpression suppressed the migration and 
invasion of ovarian cancer cells by targeting EMT-inducer ZEB1.

\section{Materials and methods}

Patients and tissue samples. The present study consisted of 20 samples of HG-SOC and 12 normal fallopian tube (FT) tissues. All samples were collected between March and June 2013 at Qilu Hospital during surgery and were immediately stored at $-80^{\circ} \mathrm{C}$. For the use of these samples, signed informed consent was obtained. All the diagnoses were confirmed by at least two pathologists.

Cell lines and culture conditions. Human ovarian cancer cell lines A2780 and SKOV3 were originally obtained from the American Type Culture Collection (ATCC, Rockville, MD, USA). Roswell Park Memorial Institute (RPMI)-1640 medium was purchased from Gibco-BRL (Rockville, MD, USA). Fetal bovine serum (FBS) was supplied by Haoyang Biological Manufacture Co. Ltd. (Tianjin, China). The human ovarian cancer cell lines A2780 and SKOV3 were cultured in RPMI-1640 medium supplemented with 10\% FBS and $100 \mathrm{U}$ penicillin-streptomycin at $37^{\circ} \mathrm{C}$ in a humidified atmosphere containing $5 \% \mathrm{CO}_{2}$.

Synthesis and transfection of miRNA mimics and miRNA inhibitors. miRNA mimics and miRNA inhibitors (2'-O-methyl modified) for in vitro transfection and its negative control (miR-nc) were designed and synthesized by RiboBio (Guangzhou, China). A2780 (3.5x10 $)$ and SKOV3 (3.0x10 $)$ cells were seeded into 6 -well plates and incubated overnight. Transfection was performed using Lipofectamine 2000 (Invitrogen, Carlsbad, CA, USA) according to the manufacturer's instructions. After $6 \mathrm{~h}$, the medium was replaced with fresh medium. During transfection, the medium was antibiotic-free.

Dual-luciferase reporter assay. The 3'-untranslated region (3'UTR) of ZEB1 mRNA containing the putative miR-1236-3p binding site was cloned into the pGL3 vector according to the manufacturer's instructions. The forward primer (5'-CGAGCT CGACAGCACAGAGCAGGAA-3') and the reverse primer (5'-CCCTCGAGTAGTTAGCACGGGTTGGA-3') were used to amplify the 3'UTR of ZEB1. The forward primer contained a $S a c 1$ restriction site and the reverse primer contained an Xho1 site. The binding site of miR-1236-3p in the ZEB1 3'UTR was mutated by using primers: F, 5'-CAGGCGCTTAAAGG AAGCTGATTAAT-3' and R, 5'-AGCGCCTGTATTGTTGC TCTCTGAGT-3'. SKOV3 cells were plated at $1.5 \times 10^{4} /$ well in a 96-well plate one day before transfection. Cells were co-transfected with $50 \mathrm{ng}$ of wild-type or mutant ZEB1 3'UTR, and 5 pmol of miR-1236-3p mimics or negative control. After $48 \mathrm{~h}$, luciferase activity was measured using the Dual-Luciferase Reporter assay system (Promega).

RNA extraction and quantitative real-time PCR. Total RNA was extracted from cells using TRIzol reagent (Invitrogen) according to the manufacturer's protocol. Then cDNA was synthesized using the PrimeScript RT reagent kit (Takara Biotechnology Co., Ltd., Dalian, China). Quantitative real-time PCR (qRT-PCR) was performed with the SYBR Premix Ex Taq
(Takara) by using the Applied Biosystems StepOnePlus ${ }^{\mathrm{TM}}$ RealTime PCR System according to the manufacturer's protocol. Glyceraldehyde-3-phosphate dehydrogenase (GAPDH) was used as the endogenous control.

Western blot analysis. The cells were washed 3 times with PBS chilled to $4^{\circ} \mathrm{C}$ and lysed on ice in RIPA buffer (Shenneng Bocai, Shanghai, China) containing protease inhibitors $(1 \mathrm{mM})$. The density of the protein was measured using the BCA protein assay kit (Merck, Darmstadt, Germany). The same amount of protein was separated by $5-10 \%$ SDS-PAGE and then transferred to a PVDF membrane (Millipore) using a semi-dry blotting apparatus (Bio-Rad Laboratories, Hercules, CA, USA). Membranes were blocked with $5 \%$ (w/v) non-fat milk in Tris-buffered saline with Tween-20 (TBST) $(100 \mathrm{mM}$ $\mathrm{NaCl}, 50 \mathrm{mM}$ Tris and $0.1 \%$ Tween-20) at room temperature for $1 \mathrm{~h}$. After the blocking step, the membranes were incubated overnight with primary antibodies (Cell Signaling Technology, Beverly, MA, USA) at $4^{\circ} \mathrm{C}$. The membranes were then washed 3 times with TBST and incubated with horseradish peroxidaselabeled secondary antibodies for $2 \mathrm{~h}$. Signals were detected with an ECL system (Merck) according to the manufacturer's instructions. GAPDH was used as the loading control.

Invasion and migration assays. Invasion assays were performed in a Transwell system (24-wells, $8-\mu \mathrm{m}$ pore size) coated with $2 \mathrm{mg} / \mathrm{ml}$ of Matrigel (both from BD Biosciences, Bedford, MA, USA). First, the cells were transfected with miR-1236-3p. After $48 \mathrm{~h}$, the transfected cells were digested with trypsin, and $1.0 \times 10^{5}$ cells were suspended in serum-free RPMI-1640 and seeded into the upper chamber of each well. The lower chamber was filled with RPMI-1640 supplemented with $20 \%$ FBS. Then the cells were fixed with formaldehyde, permeabilized with $100 \%$ methanol and stained with $0.5 \%$ crystal violet. The number of cells that had attached to the lower surface of the membrane were counted in 6 random fields under a light microscope and analyzed statistically. The cell migration assays were performed in a similar manner, except that the upper chambers were Matrigel-free.

Statistical analysis. All data are expressed as means \pm standard deviation (SD). Student's t-test (paired, 2-tail) was employed to analyze the significance of two groups. P-value $<0.05$ was considered to indicate a statistically significant result. All of the experiments were repeated at least 3 times.

\section{Results}

Expression of miR-1236-3p is downregulated in HG-SOC. To confirm the expression of miR-1236-3p in HG-SOC tissue samples, we employed qRT-PCR to quantify and compare the miR-1236-3p expression levels in the HG-SOC $(n=20)$ and FT $(n=12)$ tissue samples. As shown in Fig. 1A, the expression of miR-1236-3p was generally decreased in the HG-SOC when compared to the FT samples $(\mathrm{P}<0.001)$. This suggests that miR-1236-3p acts as a tumor suppressor in HG-SOC.

Manipulation of miR-1236-3p-induced morphological change in SKOV3 and A2780 cells. To explore the influence of miR-1236-3p on ovarian cancer cells, we transfected SKOV3 
A

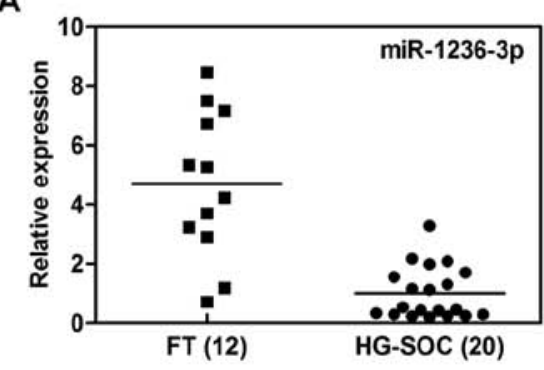

B

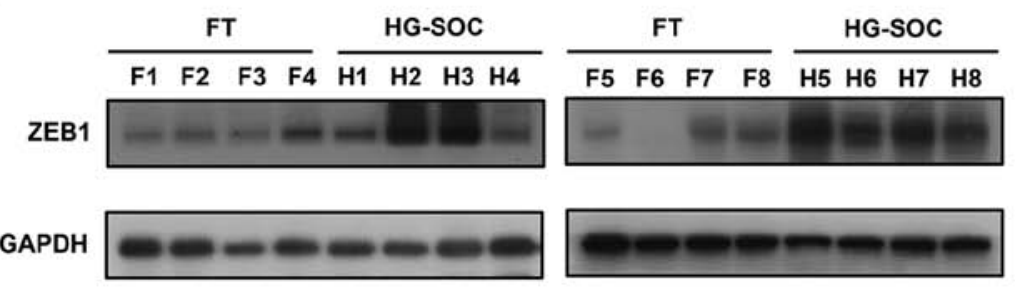

C

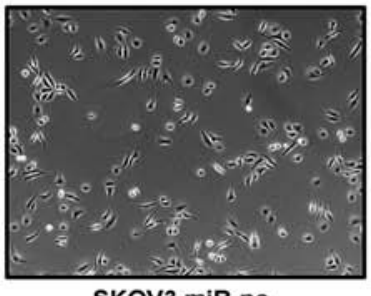

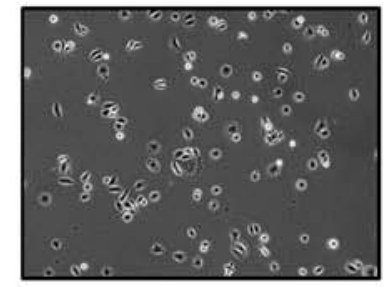

SKOV3 miR-1236-3p

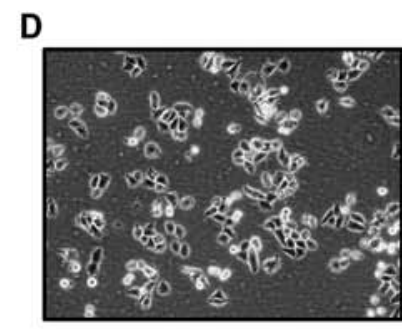

A2780 miR-nc

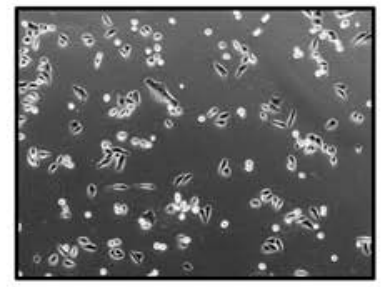

A2780 anti-miR-1236-3p

Figure 1. Expression of miR-1236-3p and ZEB1 is altered in high-grade serous carcinoma (HG-SOC), and manipulation of miR-1236-3p induces morphological changes in ovarian cancer cells. (A) qRT-PCR showed that expression of miR-1236-3p was downregulated in HG-SOC (n=20) when compared with normal fallopian tube tissue samples (FT, n=12). (B) Western blotting showed that ZEB1 expression was higher in HG-SOC $(\mathrm{n}=8)$ than that in FT tissues ( $\mathrm{n}=8$ ). (C) SKOV3 cells were transfected with miR-1236-3p mimics (SKOV3 miR-1236-3p) or negative control (SKOV3 miR-nc). After 5 days, SKOV3 miR-1236-3p cells showed a cobblestone-like appearance. (D) Spindle-like morphology was observed in the A2780 cells treated with miR-1236-3p inhibitors (A2780 antimiR-1236-3p) for 5 days. ZEB1, zinc-finger E-box binding homeobox 1.

and A2780 cells with miR-1236-3p mimics or inhibitors. As shown in Fig. 1C, SKOV3 cells treated with miR-1236-3p mimics presented a cobblestone-like appearance, while cells treated with the negative control exhibited a spindle-like morphology. We then inhibited the expression of miR-1236-3p in A2780 cells. A2780 cells transfected with the miR-1236-3p inhibitors were narrower than cells transfected with the negative control (Fig. 1D). As known, the classic morphological change correlated with EMT is the conversion from a rounded, epithelial-like form to a spindle-shaped, mesenchymal form $(8,9)$. These changes indicated that the manipulation of miR-1236-3p inhibited or stimulated the process of EMT.

Migratory and invasive abilities of ovarian cancer cells are regulated by miR-1236-3p. To further confirm the role of miR1236-3p in ovarian cancer cells, we used a two-chamber assay to evaluate the migratory and invasive capacities of SKOV3 and A2780 cells treated with miR-1236-3p mimics or inhibitors. As shown in Fig. 2, increased miR-1236-3p significantly suppressed migration and invasion of the ovarian cancer cells. In contrast, decreased miR-1236-3p promoted these abilities. Our results showed that miR-1236-3p greatly influenced migration and invasion of both ovarian cancer cell lines.

ZEB1 is targeted by miR-1236-3p. Based on the above findings, we hypothesized that miR-1236-3p regulates genes associated with EMT. Using online miRNA target prediction tools, such as microRNA.org, we found that the 3'UTR of ZEB1 mRNA contained a putative binding site for miR-1236-3p. To investigate the ability of miR-1236-3p to bind and regulate the 3'UTR of ZEB1, we performed the luciferase reporter assay. Wildtype or the mutant ZEB1 3'UTR sequence was cloned into the pGL3 vector (Fig. 3A). SKOV3 cells were co-transfected with
miR-1236-3p (mimics or negative control) and the pGL3 vector (wild-type or mutant). After $48 \mathrm{~h}$, cells were lysed to measure the luciferase activity using the Dual-Luciferase Reporter assay system. Overexpression of miR-1236-3p significantly reduced the luciferase activity in the wild-type $(\mathrm{P}<0.001)$ but not the mutant ZEB1 3'UTR (Fig. 3B). This demonstrated that ZEB1 was directly targeted by miR-1236-3p.

miR-1236-3p regulates the expression of ZEBI and EMT-related genes. We then tested whether miR-1236-3p modulates the expression of ZEB1 and EMT-related genes in ovarian cancer. First, the SKOV3 and A2780 cell lines were transfected with miR-1236-3p mimics or miR-nc. The transfected cells were analyzed by qRT-PCR and western blotting. As shown in Fig. 4A and B, ZEB1 was downregulated by miR-1236-3p mimics at both the protein and mRNA levels. We also detected the expression of EMT-related markers $\mathrm{E}$-cadherin and $\mathrm{N}$-cadherin. The results showed that the expression of E-cadherin was upregulated and the $\mathrm{N}$-cadherin expression was downregulated. Second, we downregulated the expression of miR-1236-3p in the two cell lines by using miR1236-3p inhibitors. As expected, the expression of ZEB1 and EMT-related genes showed an opposite pattern at the protein level (Fig. 4C) and mRNA level (Fig. 4D) compared to the previous assay. As known, a switch from epithelial marker E-cadherin to mesenchymal marker $\mathrm{N}$-cadherin is a classical molecular change during EMT $(8,9)$. Thus, our results indicated that miR-1236-3p may regulate the process of EMT.

Expression of ZEB1 protein is decreased in HG-SOC. Finally, we tested whether miR-1236-3p-induced ZEB1 suppression confirmed in our research was clinically relevant. As shown in Fig. 1B, we found that the expression of ZEB1 protein was 
A

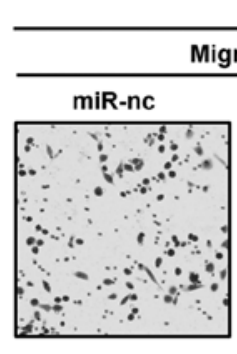

A2780

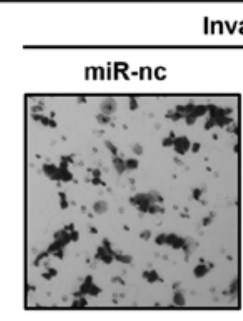

B

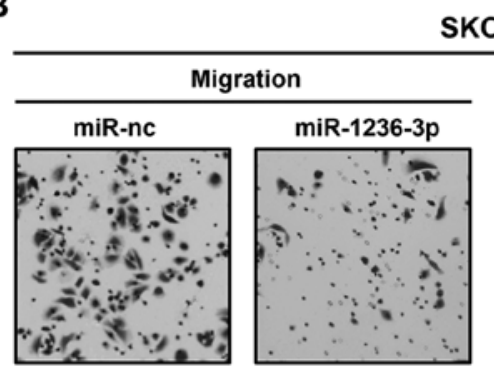

SKOV3

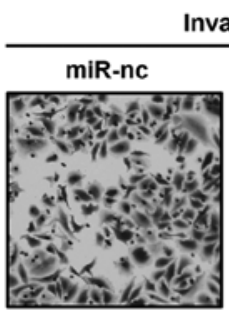

nvasion

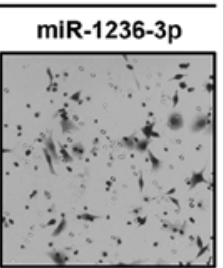

C

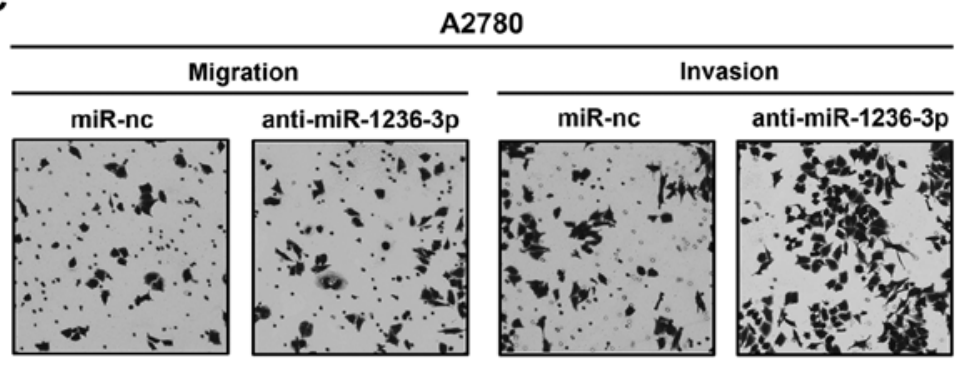

D

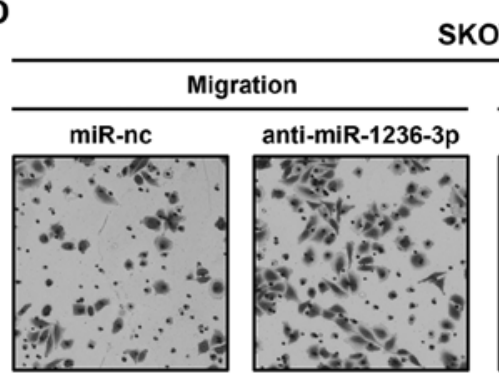

SKov3

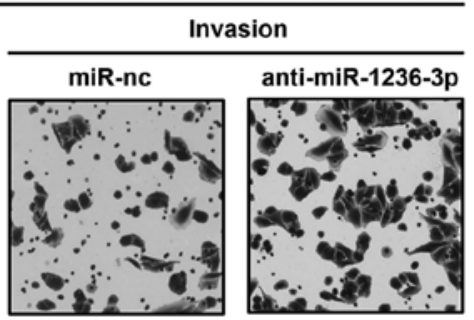

$E$

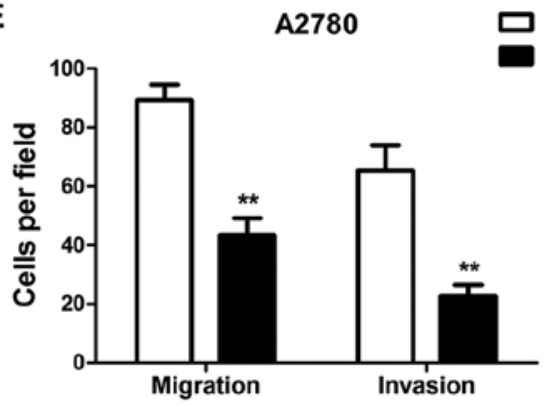

$\mathbf{F}$
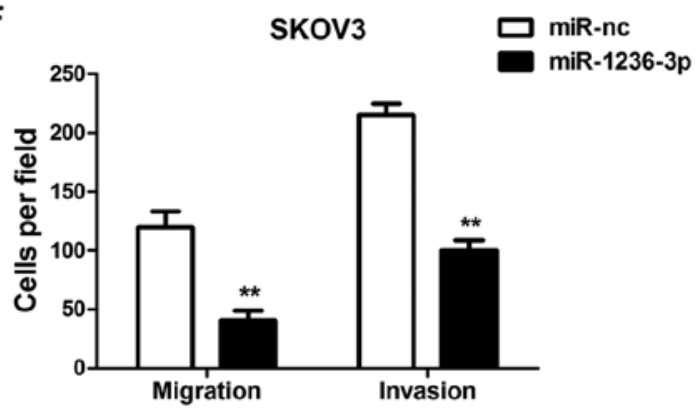

G
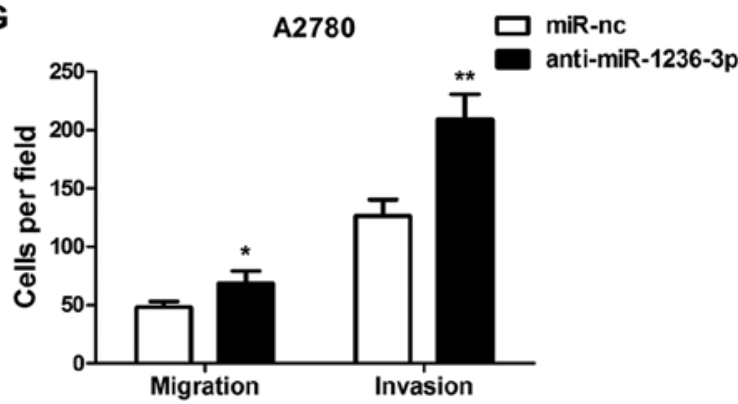

H SKOV3 吕 miR-nc

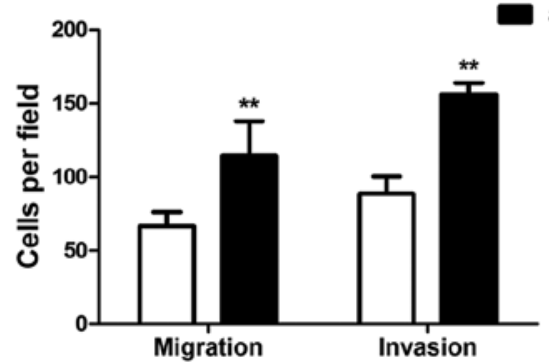

Figure 2. miR-1236-3p influences ovarian cancer cell migration and invasion. (A and B) A2780 and SKOV3 cells transfected with miR-1236-3p mimics (miR-1236-3p) showed reduced migration and invasion. (C and D) A2780 and SKOV3 cells transfected with miR-1236-3p inhibitors (anti-miR-1236-3p) showed enhanced migration and invasion. (E-H) The number of cells that migrated or invaded to the lower surface of the membrane were calculated. Data are presented as means $\pm \mathrm{SD}$. ${ }^{*} \mathrm{P}<0.05,{ }^{* *} \mathrm{P}<0.01$.

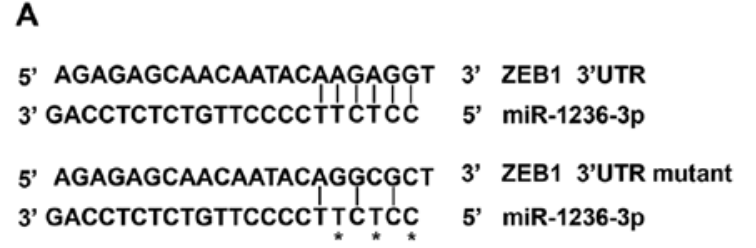

5' AGAGAGCAACAATACAAGAGGT 3' ZEB1 3'UTR 5' AGAGAgCAACAATACAgGCGCT 3' ZEB1 3'UTR mutant 3' GACCTCTCTGTTCCCCT TCTCC 5' miR-1236-3p

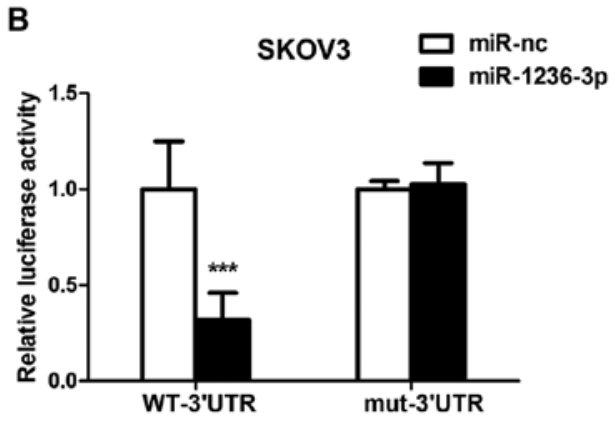

Figure 3. ZEB1 is negatively regulated by miR-1236-3p. (A) Predicted miR-1236-3p binding site in the 3'UTR of ZEB1 and mutant ZEB1 3'UTR. (B) Dual-luciferase reporter assay showed that miR-1236-3p reduced the luciferase activity in wild-type 3'UTR of ZEB1 (WT-3'UTR) but not in the mutant ZEB1 3'UTR (mut-3'UTR). Data are expressed as the means \pm SD. ${ }^{* * *} \mathrm{P}<0.001$. ZEB1, zinc-finger E-box binding homeobox 1; 3'UTR, 3'-untranslated region. 

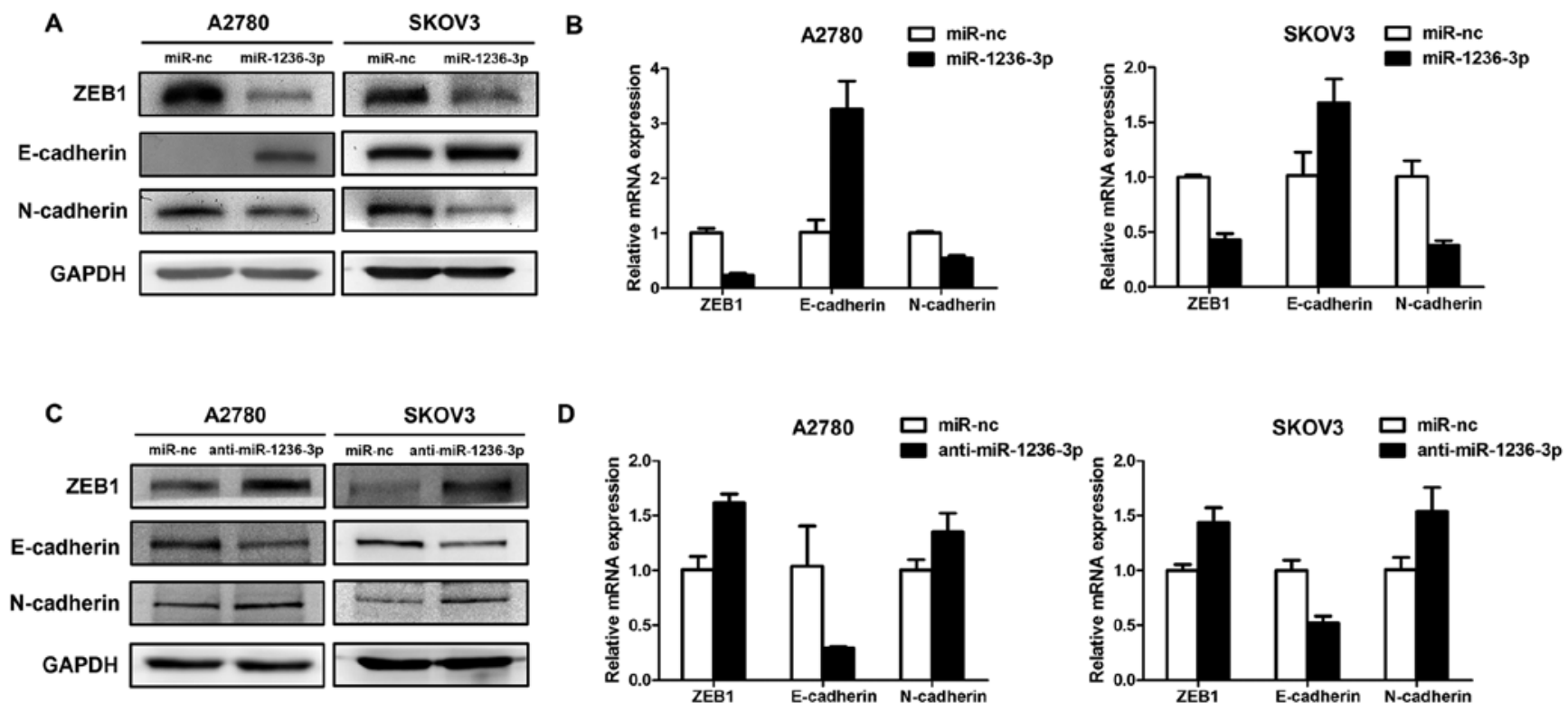

Figure 4. Alterations in the expression of ZEB1 and its downstream genes in ovarian cancer cells treated with miR-1236-3p mimics or inhibitors. (A) Western blotting and (B) qRT-PCR showed that overexpression of miR-1236-3p induced the downregulation of EMT-inducer ZEB1 and mesenchymal marker $\mathrm{N}$-cadherin, and upregulation of the epithelial marker E-cadherin. GAPDH was used as the internal control. (C) Western blotting and (D) qRT-PCR showed that cells transfected with miR-1236-3p inhibitors exhibited enhanced expression of ZEB1 and N-cadherin, and reduced expression of E-cadherin. Data are expressed as the means $\pm \mathrm{SD}$. GAPDH was used as the endogenous control. ZEB1, zinc-finger E-box binding homeobox 1; EMT, epithelial-mesenchymal transition; GAPDH, glyceraldehyde-3-phosphate dehydrogenase.

generally upregulated in HG-SOC $(\mathrm{n}=8)$ when compared to that in the FT $(\mathrm{n}=8)$ samples, and its expression was inversely correlated with miR-1236-3p. Taken together, our findings revealed that miR-1236-3p downregulation induced overexpression of ZEB1, and consequently influenced migration and invasion of HG-SOC cells.

\section{Discussion}

Early-stage ovarian cancer has few visible symptoms; therefore, most patients are diagnosed at advanced stages of disease when cancer cells have already spread (24). Current treatments (surgery, radiation and chemotherapy) are relatively ineffective for advanced ovarian cancer. Most of these patients will eventually relapse at metastatic sites. Thus, it is vital to understand the molecular mechanisms of metastasis. The present study showed that miR-1236-3p expression was decreased in HG-SOC tissue samples. Functional studies demonstrated that decreased miR-1236-3p enhanced ovarian cancer cell migration and invasion in vitro. These findings suggest that miR-1236-3p may play a potential inhibitory role in HG-SOC, and loss of miR-1236-3p may be critical for HG-SOC metastasis.

According to previous studies, miRNAs are highly tissue specific and function as tumor suppressors or oncogenes (25). The diagnostic, therapeutic and prognostic potential of miRNAs in cancer is promising (26). The miR-200 family plays an important role in the regulation of EMT by targeting the mRNA of ZEB1 and ZEB2. E-cadherin expression was also found to be correlated with miR-200 family expression in tissue samples from ovarian cancer patients $(19,20)$. miR-1236-3p is reported to be involved in the regulation of VEGFR-3 and TLR4 (27,28). Meanwhile, TLR4 and VEGFR-3 are both associated with metastasis and the poor survival of ovarian cancer patients (29-32). Therefore miR-1236-3p may be relevant to the prognosis of ovarian cancer.

EMT facilitates the ability of cancer cells to detach themselves from primary lesions, migrate to distant organs or to invade adjacent tissue, and eventually form tumor metastases. EMT is considered to be the most important step in the progression of cancer from primary tumors to other organs. Thus, blocking of EMT is an efficient approach to inhibit the spread of cancer. ZEB1 has been previously reported to be involved in cancer progression, and is known as an important transcriptional repressors of E-cadherin. In the present study, we found that miR-1236-3p may influence the process of EMT in vitro. We demonstrated that overexpression of miR-1236-3p suppressed ZEB1 expression and the motility of ovarian cancer cells, while downregulation of miR-1236-3p promoted them. Based on our findings, further studies may be required to verify the function of miR-1236-3p in vivo and whether the expression of miR-1236-3p is associated with the overall survival of HG-SOC patients.

In conclusion, the present study first demonstrated that miR-1236-3p directly targets the EMT-inducer ZEB1 and is downregulated in HG-SOC. Manipulation of miR-1236-3p regulated the invasion and migration of ovarian cancer cells. In addition, the present study indicates that miR-1236-3p may be a potential target for the prognosis and treatment of HG-SOC.

\section{Acknowledgements}

The present study was supported by the National Natural Science Foundation of China to B.K. (no. 81272857), and the National High Technology Research and Development Program of China (863 project, no. 2012AA02A507). 


\section{References}

1. Siegel R, Naishadham D and Jemal A: Cancer statistics, 2013 CA Cancer J Clin 63: 11-30, 2013.

2. Integrated genomic analyses of ovarian carcinoma. Nature 474: 609-615, 2011

3. Levanon K, Crum C and Drapkin R: New insights into the pathogenesis of serous ovarian cancer and its clinical impact. J Clin Oncol 26: 5284-5293, 2008.

4. Li J, Fadare O, Xiang L, Kong B and Zheng W: Ovarian serous carcinoma: recent concepts on its origin and carcinogenesis. J Hematol Oncol 5: 8, 2012.

5. Liu Z, Liu J, Segura MF, et al: MiR-182 overexpression in tumourigenesis of high-grade serous ovarian carcinoma. J Pathol 228: 204-215, 2012.

6. Kim J, Coffey DM, Creighton CJ, Yu Z, Hawkins SM and Matzuk MM: High-grade serous ovarian cancer arises from fallopian tube in a mouse model. Proc Natl Acad Sci USA 109 3921-3926, 2012.

7. Liu Z, Gersbach E, Zhang X, et al: miR-106a represses the RB tumor suppressor p130 to regulate cellular proliferation and differentiation in high-grade serous ovarian carcinoma. Mol Cancer Res 11: 1314-1325, 2013.

8. Thiery JP, Acloque H, Huang RY and Nieto MA: Epithelialmesenchymal transitions in development and disease. Cell 139: 871-890, 2009.

9. Vergara D, Merlot B, Lucot JP, et al: Epithelial-mesenchymal transition in ovarian cancer. Cancer Lett 291: 59-66, 2010.

10. Berx G, Raspé E, Christofori G, Thiery JP and Sleeman JP: Pre-EMTing metastasis? Recapitulation of morphogenetic processes in cancer. Clin Exp Metastasis 24: 587-597, 2007.

11. Yang G, Yuan J and Li K: EMT transcription factors: implication in osteosarcoma. Med Oncol 30: 697, 2013.

12. Spaderna S, Schmalhofer O, Wahlbuhl M, et al: The transcriptional repressor ZEB1 promotes metastasis and loss of cell polarity in cancer. Cancer Res 68: 537-544, 2008.

13. Bartel DP: MicroRNAs: target recognition and regulatory functions. Cell 136: 215-233, 2009.

14. Das E, Jana NR and Bhattacharyya NP: MicroRNA-124 targets CCNA2 and regulates cell cycle in STHdh$h^{Q 111} / H d h^{Q 111}$ cells. Biochem Biophys Res Commun 437: 217-224, 2013.

15. Nakano H, Yamada Y, Miyazawa T and Yoshida T: Gain-offunction microRNA screens identify miR-193a regulating proliferation and apoptosis in epithelial ovarian cancer cells. Int J Oncol 42: 1875-1882, 2013.

16. Icli B, Wara AK, Moslehi J, et al: MicroRNA-26a regulates pathological and physiological angiogenesis by targeting BMP/ SMAD1 signaling. Circ Res 113: 1231-1241, 2013.

17. Calin GA, Dumitru CD, Shimizu M, et al: Frequent deletions and down-regulation of micro- RNA genes miR15 and miR16 at $13 q 14$ in chronic lymphocytic leukemia. Proc Natl Acad Sci USA 99: 15524-15529, 2002.
18. Dahiya $\mathrm{N}$ and Morin PJ: MicroRNAs in ovarian carcinomas. Endocr Relat Cancer 17: F77-F89, 2010.

19. Bendoraite A, Knouf EC, Garg KS, et al: Regulation of miR-200 family microRNAs and ZEB transcription factors in ovarian cancer: evidence supporting a mesothelial-to-epithelial transition. Gynecol Oncol 116: 117-125, 2010.

20. Chen J, Wang L, Matyunina LV, Hill CG and McDonald JF: Overexpression of miR-429 induces mesenchymal-to-epithelial transition (MET) in metastatic ovarian cancer cells. Gynecol Oncol 121: 200-205, 2011.

21. Cao M, Seike M, Soeno C, et al: MiR-23a regulates TGF- $\beta$ induced epithelial-mesenchymal transition by targeting E-cadherin in lung cancer cells. Int J Oncol 41: 869-875, 2012.

22. Ru P, Steele R, Newhall P, Phillips NJ, Toth K and Ray RB: miRNA-29b suppresses prostate cancer metastasis by regulating epithelial-mesenchymal transition signaling. Mol Cancer Ther 11: 1166-1173, 2012.

23. Yokobori T, Suzuki S, Tanaka N, et al: MiR-150 is associated with poor prognosis in esophageal squamous cell carcinoma via targeting the EMT inducer ZEB1. Cancer Sci 104: 48-54, 2013.

24. Buys SS, Partridge E, Black A, et al: Effect of screening on ovarian cancer mortality: the Prostate, Lung, Colorectal and Ovarian (PLCO) Cancer Screening Randomized Controlled Trial. JAMA 305: 2295-2303, 2011.

25. Sempere LF, Christensen M, Silahtaroglu A, et al: Altered microRNA expression confined to specific epithelial cell subpopulations in breast cancer. Cancer Res 67: 11612-11620, 2007.

26. Schaefer A, Jung M, Mollenkopf HJ, et al: Diagnostic and prognostic implications of microRNA profiling in prostate carcinoma. Int J Cancer 126: 1166-1176, 2010.

27. Sato K, Yoshimura A, Kaneko T, et al: A single nucleotide polymorphism in 3'-untranslated region contributes to the regulation of Toll-like receptor 4 translation. J Biol Chem 287: 25163-25172, 2012.

28. Jones D, Li Y, He Y, Xu Z, Chen H and Min W: Mirtron microRNA-1236 inhibits VEGFR-3 signaling during inflammatory lymphangiogenesis. Arterioscler Thromb Vasc Biol 32: 633-642, 2012.

29. Yokoyama Y, Charnock-Jones DS, Licence D, et al: Vascular endothelial growth factor-D is an independent prognostic factor in epithelial ovarian carcinoma. Br J Cancer 88: 237-244, 2003.

30. Kim KH, Jo MS, Suh DS, et al: Expression and significance of the TLR4/MyD88 signaling pathway in ovarian epithelial cancers. World J Surg Oncol 10: 193, 2012.

31. Zhu Y, Huang JM, Zhang GN, Zha X and Deng BF: Prognostic significance of MyD88 expression by human epithelial ovarian carcinoma cells. J Transl Med 10: 77, 2012.

32. Klasa-Mazurkiewicz D, Jarząb M, Milczek T, Lipińska B and Emerich J: Clinical significance of VEGFR-2 and VEGFR-3 expression in ovarian cancer patients. Pol J Pathol 62: 31-40, 2011. 